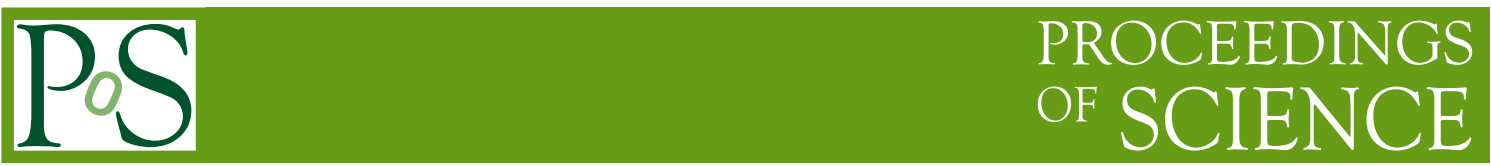

\title{
SuperKEKB status-Belle II physics run started
}

\author{
Kazunori Akai*广 \\ High Energy Accelerator Research Organization (KEK), \\ Accelerator Laboratory, \\ 1-1, Oho, Tsukuba, Ibaraki 305-0801, Japan \\ E-mail: kazunori.akai@kek.jp
}

SuperKEKB, a 7-GeV electron - 4-GeV positron double-ring collider, was constructed by upgrading KEKB to significantly push the luminosity frontier toward more detailed experiments seeking new discoveries in physics beyond the Standard Model. The design luminosity of SuperKEKB is $8 \times 10^{35} \mathrm{~cm}^{-2} \mathrm{~s}^{-1}, 40$ times higher than that achieved in KEKB. The major part of the gain results from significantly decreasing the beam sizes at the interaction point based on the nanobeam collision scheme; the design beam currents in both rings are double those achieved in KEKB. Large-scale construction for the upgrade started in 2010. After the Phase 1 beam commissioning for the upgraded rings without beam collision conducted in 2016, renovation of the interaction region, including the installation of the final-focus superconducting magnets and the Belle II detector was conducted. After completing the construction of SuperKEKB, beam tuning for collision and physics data taking by the Belle II detector started in 2018. SuperKEKB started physics run operation, pioneering luminosity frontier. In this paper, the design, construction, and beam commissioning of SuperKEKB are reviewed, mainly focusing on the accelerator.

The 39th International Conference on High Energy Physics (ICHEP2018)

4-11 July, 2018

Seoul, Korea

\footnotetext{
*Speaker.

${ }^{\dagger}$ On behalf of the SuperKEKB accelerator group
} 


\section{Introduction}

SuperKEKB is an asymmetric-energy electron-positron double-ring collider constructed by upgrading the KEKB B-Factory [1]. KEKB, which operated from December 1998 until June 2010, achieved the world's highest luminosity $\left(2.11 \times 10^{34} \mathrm{~cm}^{-2} \mathrm{~s}^{-1}\right)$; the total integrated luminosity accumulated by the Belle detector reached $1.04 \mathrm{ab}^{-1}$ [2]. Using these data, the Belle collaboration succeeded in proving the Kobayashi-Maskawa theory and obtained a variety of important experimental results in elementary particle physics. Based on the success of KEKB, upgrading to SuperKEKB [3, 4], which significantly pushes the luminosity frontier toward more detailed experiments seeking new discoveries in physics beyond the Standard Model that appears in flavor physics, is considered an urgent issue in elementary particle physics. The design luminosity of SuperKEKB is $8 \times 10^{35} \mathrm{~cm}^{-2} \mathrm{~s}^{-1}$, 40 times higher than that achieved by KEKB; our goal is to accumulate an integrated luminosity of $50 \mathrm{ab}^{-1}$.

Figure 1 shows a schematic view of the SuperKEKB collider complex. In the main ring tunnel, a 7-GeV electron ring (the high-energy ring, HER) and a $4-\mathrm{GeV}$ positron ring (the low-energy ring, LER) are aligned horizontally. The beams collide at an interaction point (IP) in the Tsukuba straight section, where the Belle II detector is located. The extremely high design luminosity of SuperKEKB required significant upgrades of the HER, LER, and final-focus system from those of KEKB. The injector Linac also required upgrading with a new positron damping ring (DR). The Belle II detector was upgraded from Belle to operate at 40 times higher physics event rates, as well as with background rates higher by a factor of 10 to 20 [5].

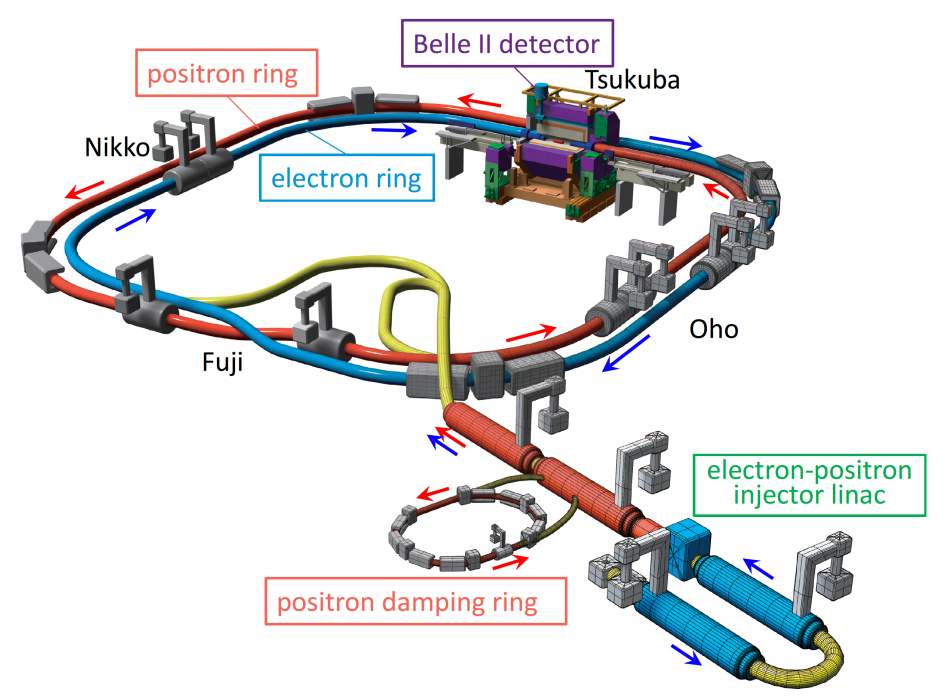

Figure 1: Schematic view of SuperKEKB. The electron and positron rings have four straight sections named Tsukuba, Oho, Fuji, and Nikko. The electron and positron beams collide at the interaction point in the Tsukuba straight section, where the Belle II detector is located. In the opposite straight section, Fuji, the two rings pass each other vertically.

The upgrade plan was approved and the construction started in 2010. After completion of the SuperKEKB construction, collision-beam tuning started in 2018, and physics data taking by Belle II started with colliding beams. After the success of factory machines including KEKB and PEP-II, 
a new era of superfactories actually began. This paper reviews the design, construction, and beam commissioning of the SuperKEKB/Belle II project, mainly focusing on the accelerator.

\section{Design overview}

For colliders with strong beam-beam effects, the luminosity is roughly proportional to the beam current $I$ and vertical beam-beam parameter $\xi_{y}$, and inversely proportional to the beta function at the IP $\beta_{y}^{*}$. In either the head-on or small-angle collision scheme, $\beta_{y}^{*}$ is limited to a value that is approximately equal to the bunch length $\sigma_{z}$; reducing $\beta_{y}^{*}$ to less than $\sigma_{z}$ does not effectively increase luminosity owing to the beam-beam force with the hourglass effect. The nanobeam collision scheme, originally proposed by P. Raimondi [6], could overcome this limitation. In the nanobeam scheme, beam bunches with sufficiently small horizontal beam size $\sigma_{x}^{*}$ collide at a large horizontal crossing angle. As longitudinal overlap of bunches is only in a small region, $\beta_{y}^{*}$ can be considerably lower than $\sigma_{z}$. SuperKEKB uses the nanobeam collision scheme to reduce $\beta_{y}^{*}$ to $0.3 \mathrm{~mm}$, which is one-twentieth that of $\operatorname{KEKB}(5.9 \mathrm{~mm})$, while $\sigma_{z}$ is approximately the same as that in KEKB. The nanobeam scheme also has an advantage that it does not require an extremely short bunch length, which mitigates parasitic loss and heating problems.

The major machine parameters of SuperKEKB are listed in Table 1 and compared with those of KEKB. The design beam currents in the LER and HER are 3.6 A and 2.6 A, respectively, which are approximately double those achieved in KEKB. The design beam-beam parameters $\xi_{y}$ for the $\mathrm{e}^{-}$and $\mathrm{e}^{+}$beams are almost the same as those of KEKB. With the reduction of $\beta_{y}^{*}$ by a factor of $1 / 20$ together with storing twice the beam currents, we can expect a luminosity 40 times higher than that of KEKB. The LER beam energy was changed from $3.5 \mathrm{GeV}$ to $4.0 \mathrm{GeV}$ to mitigate the emittance growth and short beam lifetime due to the intrabeam scattering. The HER beam energy was changed from $8.0 \mathrm{GeV}$ to $7.0 \mathrm{GeV}$ accordingly to keep the center-of-mass energy for the $\Upsilon$ resonances of the B mesons. As the ultra-low beta optics limits the size of the dynamic aperture, the beam life time is only about $6 \mathrm{~min}$ in both rings owing to the Touschek effect, radiative Bhabha scattering at the IP, beam-gas Coulomb scattering, etc, even when the energy changed to $4.0 \mathrm{GeV}$ for the LER.

\section{Upgrade construction}

The overall schedule of the SuperKEKB/Belle II project is shown in Fig. 2. Based on the discussion between the accelerator and Belle II groups, a three-phase commissioning plan was adopted. The rational of this plan was to prevent possible damage to the Belle II detectors while performing initial commissioning and tuning of the accelerator. The Phase 1 commissioning for the upgraded LER and HER was conducted before Belle II was rolled-in: low-emittance beam tuning and sufficient vacuum scrubbing were performed to improve the condition of the rings. After the installation of Belle II, Phase 2 commissioning for the beam collision tuning was conducted with the Belle II detectors but the vertex detector (VXD). The Phase 3 commissioning will start with the VXD installed, having confirmed that it can operate safely in the background situation investigated during Phase 2. 
Table 1: Machine Parameters of KEKB and SuperKEKB. Values in parentheses for SuperKEKB denote parameters without intrabeam scattering. The KEKB parameters are those achieved at the crab crossing [2], where the effective crossing angle was 0 . (The $\theta_{x}$ was $11 \mathrm{mrad}$ before the crab crossing.)

\begin{tabular}{|c|c|c|c|c|c|c|}
\hline & \multicolumn{2}{|c|}{ KEKB } & \multicolumn{2}{|c|}{ SuperKEKB } & \multirow[b]{2}{*}{ Units } \\
\hline & & $\operatorname{LER}\left(\mathrm{e}^{+}\right)$ & $\operatorname{HER}\left(\mathrm{e}^{-}\right)$ & $\operatorname{LER}\left(\mathrm{e}^{+}\right)$ & $\operatorname{HER}\left(\mathrm{e}^{-}\right)$ & \\
\hline Beam energy & $E$ & 3.5 & 8.0 & 4.0 & 7.007 & $\mathrm{GeV}$ \\
\hline Circumference & $C$ & \multicolumn{2}{|c|}{3016.262} & \multicolumn{2}{|c|}{3016.315} & $\mathrm{~m}$ \\
\hline Half crossing angle & $\theta_{x}$ & \multicolumn{2}{|c|}{0} & \multicolumn{2}{|c|}{41.5} & $\mathrm{mrad}$ \\
\hline Piwinski angle & $\phi_{\text {Piw }}$ & 0 & 0 & 24.6 & 19.3 & $\mathrm{rad}$ \\
\hline Horizontal emittance & $\varepsilon_{x}$ & 18 & 24 & $3.2(1.9)$ & $4.6(4.4)$ & $\mathrm{nm}$ \\
\hline Vertical emittance & $\varepsilon_{y}$ & 150 & 150 & 8.64 & 12.9 & $\mathrm{pm}$ \\
\hline Beta function at IP & $\beta_{x}^{*} / \beta_{y}^{*}$ & $1200 / 5.9$ & $1200 / 5.9$ & $32 / 0.27$ & $25 / 0.30$ & $\mathrm{~mm}$ \\
\hline Horizontal beam size at IP & $\sigma_{x}^{*}$ & 147 & 170 & 10.1 & 10.7 & $\mu \mathrm{m}$ \\
\hline Vertical beam size at IP & $\sigma_{y}^{*}$ & 940 & 940 & 48 & 62 & $\mathrm{~nm}$ \\
\hline Momentum compaction & $\alpha_{p}$ & 3.3 & 3.4 & 3.20 & 4.55 & $10^{-4}$ \\
\hline Energy spread & $\sigma_{\varepsilon}$ & 7.3 & 6.7 & $7.92(7.53)$ & $6.37(6.30)$ & $10^{-4}$ \\
\hline Beam current & $I$ & 1.64 & 1.19 & 3.60 & 2.60 & A \\
\hline Number of bunches & $n_{b}$ & \multicolumn{2}{|c|}{1584} & \multicolumn{2}{|c|}{2500} & \\
\hline Energy loss/turn & $U_{0}$ & 1.64 & 3.48 & 1.76 & 2.43 & $\mathrm{MeV}$ \\
\hline Long. damping time & $\tau_{z}$ & 21.5 & 23.2 & 22.8 & 29.0 & $\mathrm{~ms}$ \\
\hline RF frequency & $f_{R F}$ & \multicolumn{2}{|c|}{508.9} & \multicolumn{2}{|c|}{508.9} & $\mathrm{MHz}$ \\
\hline Total cavity voltage & $V_{c}$ & 8.0 & 13.0 & 9.4 & 15.0 & MV \\
\hline Total beam power & $P_{b}$ & $\sim 3$ & $\sim 4$ & 8.3 & 7.5 & MW \\
\hline Bunch length & $\sigma_{z}$ & $\sim 7$ & $\sim 7$ & $6.0(4.7)$ & $5.0(4.9)$ & $\mathrm{mm}$ \\
\hline Beam-beam parameter & $\xi_{x} / \xi_{y}$ & $0.127 / 0.129$ & $0.102 / 0.090$ & $0.0028 / 0.088$ & $0.0012 / 0.081$ & \\
\hline $\begin{array}{l}\text { Luminosity } \\
\text { Integrated luminosity }\end{array}$ & $\begin{array}{l}L \\
\int L\end{array}$ & \multicolumn{2}{|c|}{$\begin{array}{c}2.108 \times 10^{34} \\
1.041\end{array}$} & \multicolumn{2}{|c|}{$\begin{array}{c}8 \times 10^{35} \\
50\end{array}$} & $\begin{array}{c}\mathrm{cm}^{-2} \mathrm{~s}^{-1} \\
\mathrm{ab}^{-1}\end{array}$ \\
\hline
\end{tabular}

The upgrade construction was optimized to adjust to the phased commissioning plan. The construction of the LER and HER was completed in 5.5 years before Phase 1. After Phase 1, the renovation work of the interaction region (IR) was conducted; a new final-focus superconducting magnet system (QCS) and the Belle II detector were installed for Phase 2. The DR construction was completed before Phase 2, when it is indispensable (DR was not needed for Phase 1). The Linac upgrade proceeded step by step to meet the injection-beam quality required at each phase, while operating for photon factory rings.

\subsection{Main rings}

To upgrade KEKB to SuperKEKB, new equipment was employed, including products developed to meet the challenging requirements for SuperKEKB, whereas the existing tunnel, infrastructure, and accelerator components for KEKB were reused wherever possible. The KEKB lattice structure in the arc sections consisting of $2.5 \pi$ unit cells were preserved as much as possible by utilizing their wide tuning ranges on both the horizontal emittance $\varepsilon_{x}$ and the momentum compaction factor $\alpha_{p}$. To achieve the design value of $\varepsilon_{x}$ in the LER, the main dipole magnets were replaced 


\section{SuperKEKB/Belle II schedule}

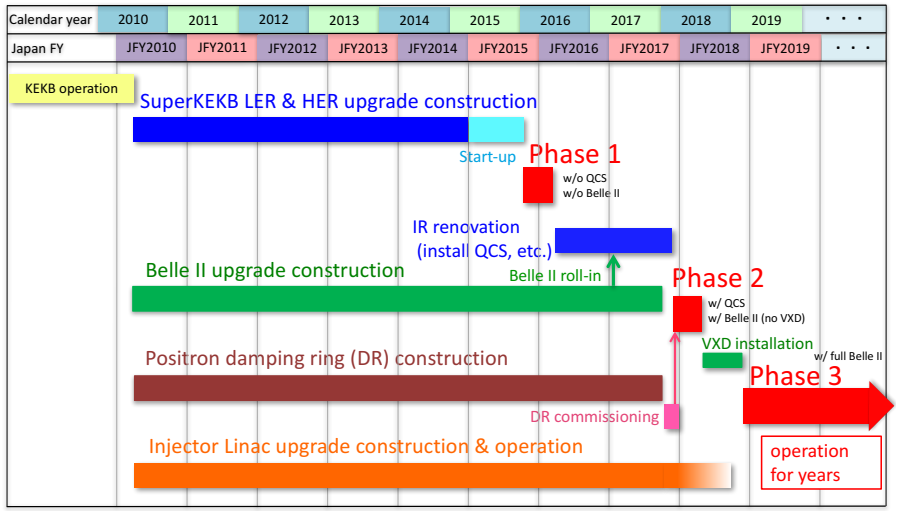

Figure 2: Overall schedule of SuperKEKB/Belle II project.

with longer ones, and the wiggler sections were reformed to have twice as many wiggle pitches by adding new magnets. The arc sections in the HER were reused because $\varepsilon_{x}$ could be decreased acceptably by adjusting the quadrupole magnets in the $2.5 \pi$ cells. To further reduce $\varepsilon_{x}$, a new wiggler section was built in the HER by utilizing some of the reused wiggler magnets of the LER.

For the nanobeam collision scheme with an extremely low $\beta_{y}^{*}$, a new QCS was developed, which required state-of-the-art design and technology (see Sec. 3.2). The beam lines for the $\sim 300$ $\mathrm{m}$ around the final-focus sections in both rings were fully reconstructed to incorporate local chromaticity correction sections for both the vertical and horizontal planes to correct the large chromaticity due to small values of $\beta_{x}^{*}$ and $\beta_{y}^{*}$ as well as to fit the new collision scheme with a large crossing angle within the existing tunnel.

To cope with the electron cloud effect (ECE) in the LER, countermeasures stronger than ever are required for SuperKEKB. Beam pipes in most of the LER were replaced with new antechambertype pipes with a TiN coating inside. Various other measures such as using groove-shaped surfaces in the dipole magnets, clearing electrodes in the wiggler magnets, and solenoids in field-free regions were also taken.

To increase beam currents to twice that achieved in KEKB, vacuum components were upgraded for lower impedance and higher thermal strength in addition to the countermeasures taken to address the ECE. The radio frequency (RF) system was rearranged and reinforced for delivering higher power to the beam, with normal-conducting cavities composed of three-cavity system called ARES (Accelerator Resonantly coupled with Energy Storage) and superconducting cavities reused after necessary improvements were taken to their input couplers and higher-order mode dampers. With this reinforcement approximately $70 \%$ of the design beam currents in both rings can be stored. Further reinforcement of the RF system is foreseen during Phase 3, depending on beam condition requirements and budgeting. Figure 3 shows straight and arc sections of SuperKEKB main rings with the magnets, beam pipes and RF cavities.

The beam instrumentation systems were upgraded to provide the higher performance required 
for lower emittance beams and smaller beam size. Various components were developed under collaborations, including bunch-by-bunch transverse feedback systems, longitudinal feedback system with overdamped kickers, X-ray beam-size monitors, a fast luminosity monitor, and a large-angle beamstrahlung monitor.
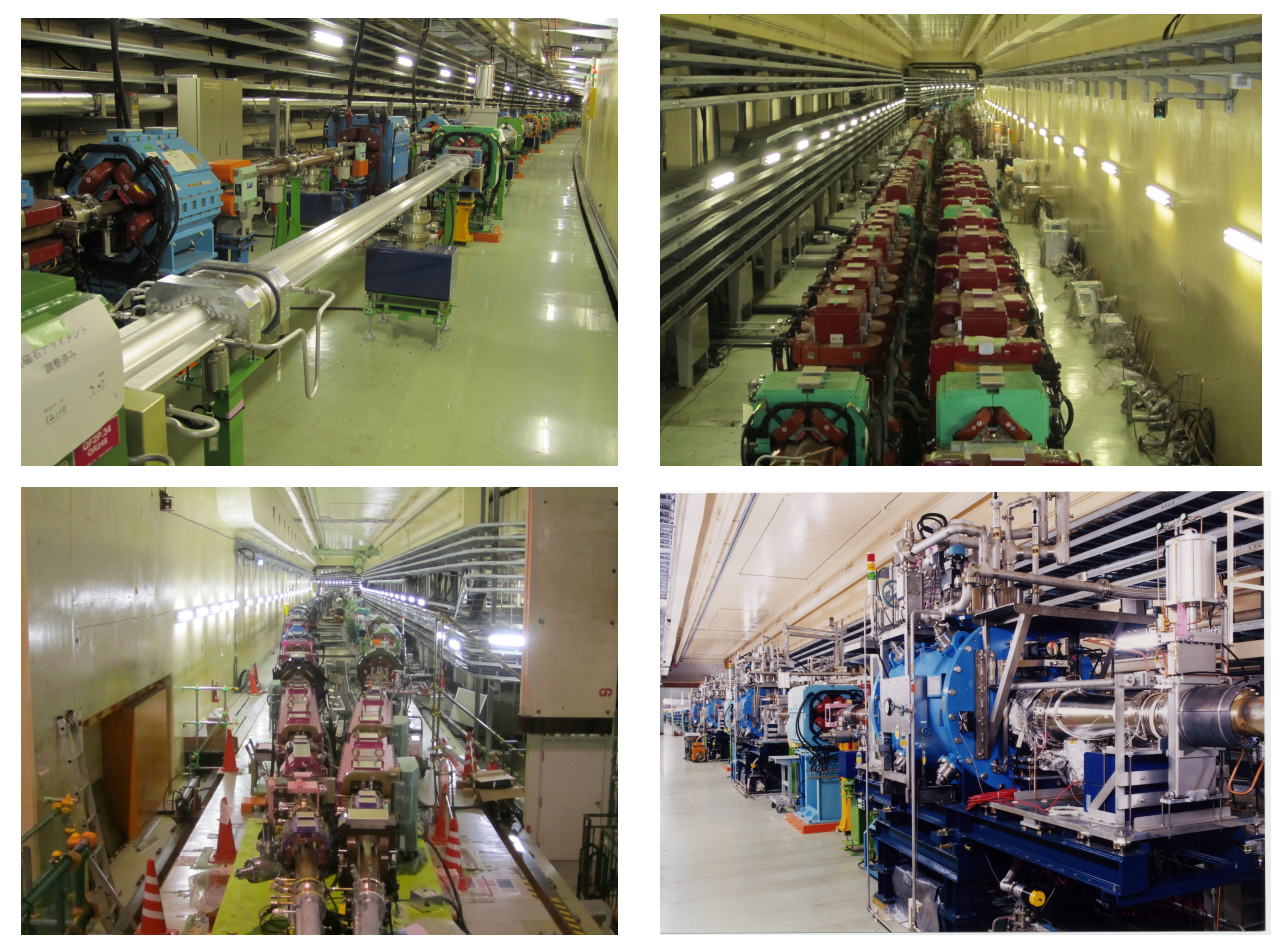

Figure 3: SuperKEKB main rings: (upper left) antechamber-type beam pipes for the LER in an arc section, (upper right) reinforced wiggler sections for the LER and HER with the ARES cavities towards the back, (lower left) new beam line on the left side of the IP, and (lower right) superconducting cavities in the HER.

\subsection{IR renovation for Phase 2}

After completing the construction of LER and HER except for the IR, the Phase 1 commissioning without beam collision was conducted in 2016 (see Sec. 4.1). After Phase 1 was completed, the IR renovation work was conducted to install the QCS and the Belle II detector. The new QCS for SuperKEKB is composed of eight main quadrupoles, solenoid coils to cancel the magnetic field of Belle II, and more than 40 corrector coils. These coils are accommodated in two cryostats, QCSL and QCS-R, located on both sides of the IP. Many stringent requirements were imposed on the QCS: independent quadrupoles for each beam line to be located as close to the IP as possible, complicated design to meet optics requirements, significant space constraints owing to the boundary with the Belle II components as well as being very close to the beam pipes at room temperature, etc. Corrector coil windings were done under collaboration with BNL and a single-stretched wire alignment system was introduced under collaboration with FNAL. After their successful fabrication, the QCS-L and QCS-R were installed in July 2016 and February 2017, respectively (Fig. 4, left). Magnetic field measurement of the QCS on the beam line was conducted from May to August 
in 2017; the results showed sufficiently good performance for Phase 2 operation in terms of both the field quality and alignment of the magnets.

While the mechanical structure and superconducting solenoid magnet of Belle were reused, all the detector components for Belle II were required to be either new or considerably upgraded for higher physics event rates and background rates. Details of the upgrade of each sub-detector are reported elsewhere [5]. The new VXD consists of a Pixel Vertex Detector (PXD) based on DEpleted P-channel Field Effect Transistor (DEPFET) technology and Silicon strip Vertex Detector (SVD). The Central Drift Chamber (CDC), which surrounds the VXD, was upgraded with smaller cells and longer lever arms. A particle identification system includes the Time-Of-Propagation (TOP) system in the barrel region and Aerogel Ring Image CHerenkov (ARICH) in the forward region. The Electromagnetic CaLorimeter (ECL) and the $K_{L}$-muon (KLM) detector were also improved for higher hit rate and neutron background. The installation of sub-detectors proceeded mostly starting from the outer to the inner detectors in the following order: KLM, TOP, CDC, and the backward ECL. The Belle II was moved to the IP in beam line (roll-in) in April 2017. After the field measurement of the QCS, the forward detectors, ARICH and end-cap ECL, were added from August to November 2017. Finally, the QCS was moved toward the IP to be connected to the IP chamber of Belle II (Fig. 4, right), and the IR renovation work for Phase 2 was completed in March 2018.
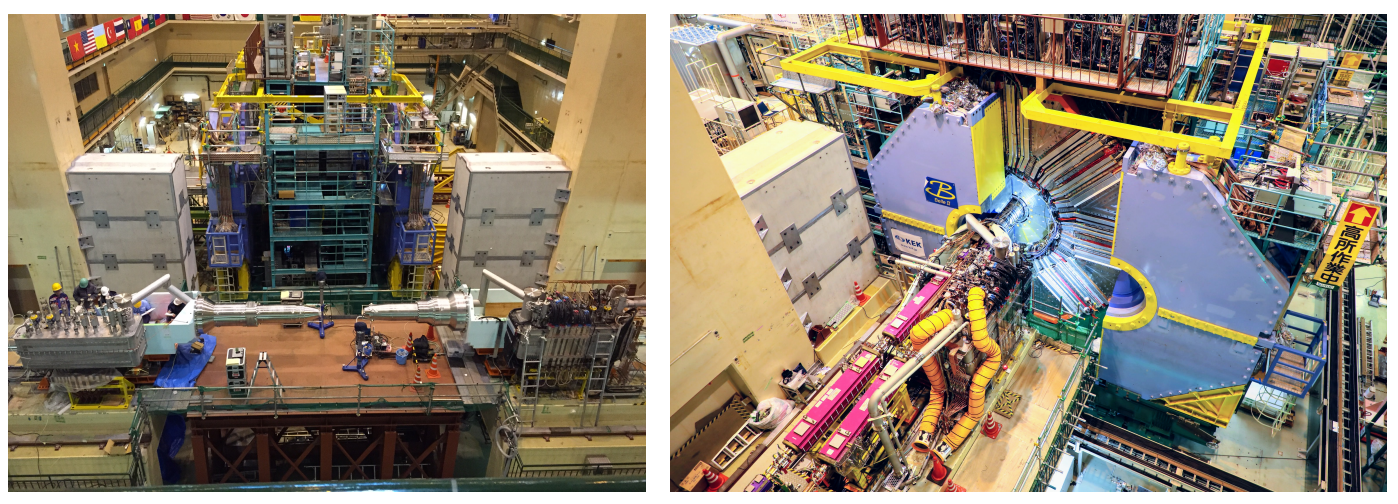

Figure 4: IR of SuperKEKB: (left) QCS-L and QCS-R installed on both sides of the IP. The Belle II stays out of the beam line then (Feb. 2017), and (right) the QCS moved forward to the IP and connected to the IP chamber of Belle II (Jan. 2018).

\subsection{Injector complex}

The injector Linac was upgraded to satisfy the requirements of injection beams with higher current and lower emittance. For the electron beam, a new low-emittance RF electron gun was developed, which was successfully operated in Phase 2. For the positron beam, the positron source was improved with a flux concentrator. A new $1.1 \mathrm{GeV}$ positron DR was designed and built to reduce positron beam emittance before injection into the LER. As the Linac delivers beams not only to SuperKEKB, but also to photon factory rings (PF and PF-AR), improving the simultaneous top-up injections into these rings is crucial for extremely short beam life time of the LER and HER. To change the pulse-by-pulse beam optics in the Linac adjusted for each ring, pulse steering and quad magnets were installed. 


\section{Commissioning}

\subsection{Phase 1 commissioning}

The Phase 1 commissioning for the upgraded LER and HER was conducted from February to June 2016 without beam collision. The purposes of Phase 1 were to perform basic machine tuning, low emittance beam tuning, vacuum scrubbing with beam currents up to approximately $1 \mathrm{~A}$, beam background study, etc. The vertical emittance $\varepsilon_{y}$ of around $10 \mathrm{pm}$ was achieved in both rings, as was confirmed by both beam size measurement and evaluation from optical functions. The beam currents were successfully increased up to $1.01 \mathrm{~A}$ in the LER and $0.87 \mathrm{~A}$ in the HER. The beam doses of $780 \mathrm{Ah}$ and $660 \mathrm{Ah}$ were achieved in the LER and HER, respectively, which exceeded the requirement by the Belle II group. The details of the Phase 1 commissioning are reported elsewhere [7, 8].

\subsection{Phase 2 commissioning}

Having completed the renovation work of the IR, including installation of the QCS system and the Belle II detector, Phase 2 commissioning started in March 2018. The main purposes of Phase 2 are (1) verification of the nanobeam collision scheme, achieving luminosity of around $10^{34} \mathrm{~cm}^{-2} \mathrm{~s}^{-1}$, and having a clear path for further improvement; and (2) to examine the background to verify that the VXD can be installed at the center of Belle II for Phase 3.

The beam tuning began with detuned optics, which has no significant squeezing at the IP, to establish stable injection and beam storage in the HER and LER with the new IR and DR. The HER injection tuning started on 19 March and beam storage was achieved on 21 March. The LER injection tuning started on 26 March and beam storage was successful on 31 March. Subsequently, beam tuning with squeezing beta was conducted. After stable storage with $\beta_{x}^{*}=200 \mathrm{~mm}$ and $\beta_{y}^{*}=8$ $\mathrm{mm}$ was established for each ring, collision tuning was performed. Collision point and timing were adjusted by shifting the bunch bucket, scanning RF phase, and the vertical orbit at the IP. Having confirmed stable beam collision, the Belle II detectors were switched on, and the first hadronic event (Fig. 5) was observed on 26 April. This was the first collision in a new collider in the world that took place for 10 years after LHC.

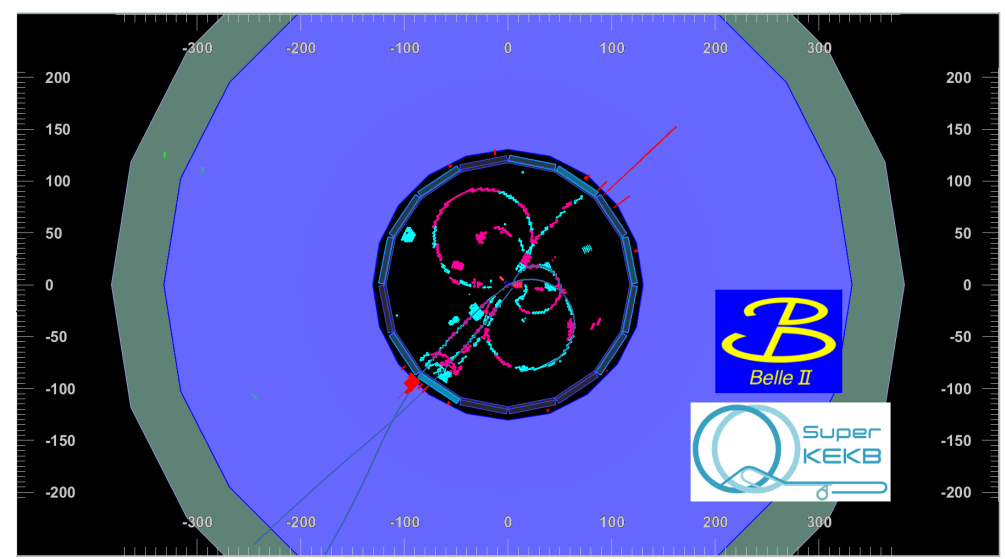

Figure 5: First hadronic event observed by Belle II. 
Further squeezing the betas, mainly $\beta_{y}^{*}$ and partly $\beta_{x}^{*}$, was performed step by step. On 23 May, luminosity exceeded $10^{33} \mathrm{~cm}^{-2} \mathrm{~s}^{-1}$ with $\beta_{y}^{*}=6 \mathrm{~mm}(350 \mathrm{~mA} / 290 \mathrm{~mA}$ in LER/HER with 789 bunches). After $\beta_{y}^{*}$ was further squeezed to $4 \mathrm{~mm}$, we struggled in increasing luminosity for about three weeks. The observed specific luminosity at low bunch current was much lower than that estimated from the beam size measurement, which suggested that some geometrical mismatch exists between the two beams at the IP. Then, it was solved by searching for a much wider range of $x-y$ coupling parameters at the IP. It is suspected that a considerably large unknown $x-y$ coupling source may exist around the IP.

By continuously improving the beam tuning, the peak luminosity achieved during Phase 2 was $5.55 \times 10^{33} \mathrm{~cm}^{-2} \mathrm{~s}^{-1}$ with $\beta_{y}^{*}=3 \mathrm{~mm}\left(788 \mathrm{~mA} / 778 \mathrm{~mA}, N_{b}=1576\right)$. The specific luminosity reached $2 \times 10^{31} \mathrm{~cm}^{-2} \mathrm{~s}^{-1} /(\mathrm{mA})^{2}$, the target value for Phase 2 . Collision at higher bunch currents with smaller number of bunches was also performed, and the maximum luminosity in this operation mode was $2.29 \times 10^{33} \mathrm{~cm}^{-2} \mathrm{~s}^{-1}$ at $265 \mathrm{~mA} / 217 \mathrm{~mA}$ with $N_{b}=395$. With four times bunches $\left(N_{b} \sim\right.$ 1576) the same collision condition might give $\sim 9 \times 10^{33} \mathrm{~cm}^{-2} \mathrm{~s}^{-1}$, assuming no degradation due to multi-bunch effects. The details of Phase 2 performance are presented elsewhere $[9,10]$.

The beam background to the Belle II detectors was sensitive to machine operation conditions such as beam optics, collision orbit, injection conditions, etc. Continuous efforts for improving beam conditions as well as fine tuning of collimator settings were made to improve the background. In the innermost space of Belle II, where VXD is to be installed for Phase 3, BEAST2 (Beam Exorcism for A Stable Belle II Experiment) was installed for measuring the background rates and operating conditions during Phase 2. The observation by these sensors during the beam operation confirmed that the VXD can be installed and operated safely to start Phase 3.

Data taking and analysis in Belle II group is being performed steadily. Some of the preliminary data using the integrated luminosity of $250 \mathrm{pb}^{-1}$ are presented in this conference by colleagues of the Belle II group. The rediscovery of charm-reconstructed mass for $\mathrm{D}^{*}$ and the mass for $\pi$ -

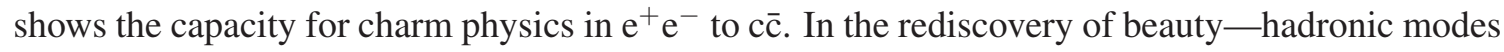
and semileptonic decay modes from $\mathrm{b}$ to $\mathrm{c}$ - the peak and width of the signal in beam-energy constrained mass is consistent with Monte-Carlo.

\subsection{Toward Phase 3}

By evaluating the progress during Phase 2, in particular the luminosity performance and background situation for the VXD installation, it was decided to complete Phase 2 by mid-July 2018 as scheduled and to start transition work toward Phase 3. The VXD will be installed in Belle II, and the Phase 3 commissioning is scheduled to start early 2019. In Phase 3, full-fledged physics run will continue with increasing luminosity by further squeezing the betas and increasing the beam currents.

An issue encountered during Phase 2 was frequent quenches of the QCS due to unexpected beam loss. Several measures were taken, including appropriate adjustment of the collimator settings and introducing beam abort function by Belle II diamond sensor signals. Continuous efforts to improve beam injection and more careful operation of experience were also made. Although these measures effectively reduced the frequency of the quenches, the number of quenches increased again as the beam current was increased. One concern was that the number of collimators seemed insufficient, in particular for the LER. Four horizontal (H-) collimators and one vertical (V-) one 
for the antechamber beam pipes, developed based on those used in PEP II at SLAC, were used in the LER during Phase 2. In the design consideration, from the viewpoint of transverse mode coupling instability, the number of V-collimator in the LER was limited to only one. In the Phase 2 operation, however, it turned out that more collimators are necessary to avoid QCS quenches and to reduce beam background. In order to mitigate the problem, five collimators are under fabrication (three H- and one V-for LER, one H- for HER) to be added by the start of Phase 3. In the HER, on the other hand, more collimators exist, including reused ones attached on the reused beam pipes in the arc sections.

\section{Summary}

A large-scale construction of SuperKEKB by upgrading KEKB was completed, and collision beam tuning and physics data taking by the Belle II detector began in Phase 2 commissioning. The luminosity performance with the nanobeam collision scheme was experimentally verified for the first time in a real collider with a large Piwinski angle. A new era of collision experiment started with upgraded SuperKEKB and Belle II, pioneering a luminosity frontier. In Phase 3, which will start in 2019 with full Belle II detectors installed, SuperKEKB will promote development of luminosity frontier for unprecedented area by continuously improving machine performance to provide a large quantity of collision data to Belle II required for physics discovery beyond the Standard Model.

\section{References}

[1] KEKB B-Factory Design Report, KEK Report 95-7 (1995).

[2] T. Abe et al., Achievements of KEKB, Prog. Theor. Exp. Phys. 2013 (2013) 03A001.

[3] Y. Ohnishi et al., Accelerator Design at SuperKEKB, Prog. Theor. Exp. Phys. 2013 (2013) 03A011.

[4] Preliminary version of Technical Design Report of SuperKEKB, https://kds.kek.jp/indico/event/15914/.

[5] T. Abe et al., Belle II Technical Design Report, 2010, arXiv:1011.0352.

[6] SuperB Conceptual Design Report, INFN/AE-07/2, SLAC-R-856, LAL 07-15 (March 2007).

[7] Y. Ohnishi, Start of SuperKEKB, in Proceedings of ICHEP2016, Chicago, USA (2016) 006.

[8] Y. Funakoshi, Commissioning of SuperKEKB, in Proceedings of eeFACT2016, Daresbury, UK (2016) 4.

[9] Y. Ohnishi, Report on SuperKEKB Phase 2 Commissioning, in Proceedings of IPAC2018, Vancouver, BC, Canada (2018) 1.

[10] A. Morita, Status of SuperKEKB Phase 2 Commissioning, this conference. 